\title{
Invariance of generalized wordlength patterns
}

\author{
Jay H. Beder \\ Jeb F. Willenbring \\ Department of Mathematical Sciences \\ University of Wisconsin-Milwaukee \\ P.O. Box 413 \\ Milwaukee, WI 53201-0413 \\ beder@uwm.edu, jw@uwm.edu
}

\begin{abstract}
The generalized wordlength pattern (GWLP) introduced by Xu and Wu (2001) for an arbitrary fractional factorial design allows one to extend the use of the minimum aberration criterion to such designs. Ai and Zhang (2004) defined the $J$-characteristics of a design and showed that they uniquely determine the design. While both the GWLP and the $J$-characteristics require indexing the levels of each factor by a cyclic group, we see that the definitions carry over with appropriate changes if instead one uses an arbitrary abelian group. This means that the original definitions rest on an arbitrary choice of group structure. We show that the GWLP of a design is independent of this choice, but that the $J$-characteristics are not. We briefly discuss some implications of these results.
\end{abstract}

Key words. Fractional factorial design; group character; Hamming weight; multiset; orthogonal array AMS(MOS) subject classification. Primary: 62K15; Secondary: 05B15, 15A69, 20C15, $62 \mathrm{~K} 05$

\section{Introduction}

In a regular fractional factorial design $D$, the quantities

$$
A_{i}(D)=\text { the number of defining words of length } i
$$

contain useful information about the design. In particular, the smallest index $i$ for which $A_{i}(D)>0$ is the resolution of the design. Moreover, one way of comparing two designs having $k$ factors and equal resolution is to compare their wordlength patterns $\left(A_{1}, A_{2}, \ldots, A_{k}\right)[6,7]$. The better design is said to have less aberration.

While nonregular designs no longer have defining words as such, a generalized wordlength pattern $(G W L P)$ can be defined for them combinatorially. This was done for two-level designs by Tang and Deng [13], and was generalized to arbitrary (possibly mixed-level) designs by Xu and $\mathrm{Wu}[15]$ using group characters.

An intermediate computation in the two-level case gives a set of values that Tang and Deng called $J$-characteristics (first introduced in [4]), and Tang [12] showed that these numbers completely determine the design $D$, somewhat analogous to the way that a defining subgroup determines a regular design. Ai and Zhang [1] generalized this to arbitrary designs by looking closely at the corresponding computation in [15]. 
In defining generalized wordlength patterns of arbitrary designs, $\mathrm{Xu}$ and $\mathrm{Wu}[15]$ assigned to the $i$ th factor the cyclic group $\mathbb{Z}_{s_{i}}$, where $s_{i}=$ the number of levels of the factor. While this choice is a computational convenience, it is also arbitrary, and in fact the calculation of the GWLP can be carried through using other abelian groups as well, as we indicate below.

This, however, raises the following question for non-prime $s_{i}$. Since the (irreducible) characters of two groups of equal order will generally be different, does the choice of group affect either the $J$-characteristics or the GWLP of a given design? Certainly any dependence of the GWLP on an arbitrary choice would raise a serious question about its use in comparing designs using relative aberration. It will be clearly seen that the $J$-characteristics do depend on this choice. However, perhaps surprisingly, this does not affect the values of the GWLP. That is our main result.

There are many excellent expositions of character theory, such as [8, [9] and [10. In general we will mention known results without citation. We will also use a number of results from multilinear algebra (the theory of tensor products). These are collected in an appendix.

Notation. We will denote the integers by $\mathbb{Z}$, and the integers modulo $s$ by $\mathbb{Z}_{s}$ as above. The complex numbers will be denoted by $\mathbb{C}$ and complex Euclidean space by $\mathbb{C}^{s}$. Vectors in $\mathbb{C}^{s}$ will be viewed as columns. The conjugate of $z \in \mathbb{C}$ will be denoted by $\bar{z}$, the transpose of a vector or matrix by a prime $\left({ }^{\prime}\right)$, and the adjoint (or conjugate transpose) of a matrix or linear transformation $A$ by $A^{*}$. The inner product of $v=\left[v_{1}, \ldots, v_{s}\right]^{\prime}$ and $w=\left[w_{1}, \ldots, w_{s}\right]^{\prime} \in \mathbb{C}^{s}$ is given by

$$
\langle v, w\rangle=\sum_{i=1}^{s} v_{i} \bar{w}_{i} .
$$

The cardinality of a set $E$ will be written $|E|$.

The Hamming weight of $u=\left(u_{1}, \ldots, u_{k}\right)$, wt $(u)$, is the number of nonzero components of $u$. (In Section 2 we will replace "nonzero" by "nonidentity" in order to deal with groups whose identity element is not 0 .)

We alert the reader to the fact that we will use $G$ (or $G_{i}$ ) as an index set, with elements $g$ or $h$. Sometimes such sets will be groups, but often they will be viewed just as sets. We will try to make absolutely clear from context when a result requires a group structure and when it doesn't.

\section{Definitions}

A fractional factorial design on $k$ factors is a multisubset $D$ of a finite Cartesian product $G=G_{1} \times \cdots \times G_{k}$, that is, the set $G$ with the element $g$ repeated $O(g)$ times, $O(g) \geq 0$. The set $G_{i}$ indexes the $s_{i}$ levels of factor $i$, and we let $s=s_{1} \cdots s_{k}$. We will refer to $O$ as the counting or multiplicity function of $D$. The elements ( $k$-tuples) of the design are referred to as runs, and the number of runs in the design, counting multiplicities, is

$$
N=|D|=\sum_{g \in G} O(g)
$$


The design $D$ may also be viewed as an orthogonal array, particularly if its runs are displayed in matrix form, say as columns of a $k \times N$ matrix.

In [15] Xu and Wu defined the generalized wordlength pattern $\left(A_{1}(D), \ldots, A_{k}(D)\right)$ of $D$ as follows. If $G_{i}$ has $s_{i}$ elements, we take $G_{i}=\mathbb{Z}_{s_{i}}$, the additive group of integers modulo $s_{i}$. This makes $G$ an abelian group. To each $g \in \mathbb{Z}_{s}$ we associate a function $\chi_{g}: \mathbb{Z}_{s} \rightarrow \mathbb{C}$ such that

$$
\chi_{g}(h)=\xi^{g h}
$$

where $\xi$ is a primitive $s$ th root of unity (say $\left.\xi=e^{2 \pi i / s}\right)$. For elements $g=\left(g_{1}, \ldots, g_{k}\right.$ ) and $h=\left(h_{1}, \ldots, h_{k}\right)$ of $G=G_{1} \times \cdots \times G_{k}$, we let

$$
\chi_{g}(h)=\prod_{i} \chi_{g_{i}}\left(h_{i}\right)
$$

and define 1

$$
\chi_{g}(D)=\sum_{h \in G} O(h) \chi_{g}(h) .
$$

Finally, the "generalized wordlengths" are given by

$$
A_{j}(D)=N^{-2} \sum_{\operatorname{wt}(g)=j}\left|\chi_{g}(D)\right|^{2} \quad \text { for } j=1, \ldots, k,
$$

where $\operatorname{wt}(g)$ is the Hamming weight of $g$.

$\mathrm{Ai}$ and Zhang [1] note that when $s_{1}=\cdots=s_{k}=2$ the quantities $\chi_{g}(D)$ are the $J$ characteristics of Tang and Deng, and rename them so in the general case, with the notation $J_{g}(D)$.

We now indicate the way in which other groups may be used in (5) and (6).

The functions $\chi_{g_{i}}$ are the irreducible characters of the group $\mathbb{Z}_{s_{i}}$, and so the functions $\chi_{g}$ are the irreducible characters of $G$. Among these is $\chi_{e} \equiv 1$, the trivial character of $G$, corresponding to the identity $e$ of $G$. Something similar holds for abelian groups, in particular the indexing of irreducible characters by group elements.

Specifically, the irreducible characters of an abelian group $G$ are precisely the homomorphisms of $G$ into the multiplicative group $\mathbb{C}^{*}=\mathbb{C} \backslash 0$. The indexing of these characters is based on the following result.

Theorem 2.1. Let $\operatorname{Irr}(G)$ denote the set of irreducible characters of the group $G$. If $G$ is abelian, then $\operatorname{Irr}(G)$ forms a group under pointwise multiplication, and if $G$ is also finite, then $G \cong \operatorname{Irr}(G)$. In particular, the identity element of $G$ corresponds to the trivial character of $G$.

The isomorphism is not canonical - and, in particular, not unique - as it depends on the representation of an abelian group as a product of cyclic groups (the Fundamental Theorem of Abelian Groups), and for cyclic groups on the choice of root of unity in (3). (See, e.g., [9, Theorem 2.4]). We will assume that we have fixed an isomorphism $G_{i} \rightarrow \operatorname{Irr}\left(G_{i}\right)$ for each $i$, and thus an indexing of the irreducible characters of $G_{i}$ by group elements. We will not need

\footnotetext{
${ }^{1}$ In [15] $\chi_{g}(D)$ is defined as $\sum_{h \in D} \chi_{g}(h)$, and it is to be understood that the $h$ th term is repeated the number of times $h$ appears in the design [14]. Equation (5), which is essentially the same as that used in [1, makes this explicit.
} 
to know the indexing explicitly. The irreducible characters of the direct product $G$ are still given by (41).

$J$-characteristics and generalized wordlength counts are still defined by (5) and (6), respectively, where we now define the weight of the element $g=\left(g_{1}, \ldots, g_{k}\right) \in G$ to be the number of nonidentity components of $g$. Our main result is this:

Theorem 2.2. The quantities $A_{j}(D)$ in (6) are independent of the group structure of $G$.

The proof of this theorem is given in Section 4, Before considering this, we take a moment to study the effect of the choice of group on the $J$-characteristics of a design.

\section{J-characteristics. The character table.}

We see that the irreducible characters of a finite abelian group $G$ of order $s$ may be written $\chi_{g_{1}}, \ldots, \chi_{g_{s}}$, where $g_{i}$ are the elements of $G$ in some order. The values $\chi_{g_{i}}\left(g_{j}\right)$ form the character table of $G$, the columns of which are mutually orthogonal and of norm $\sqrt{s}$ (with respect to the inner product (1)). Another way to say this is that the $s \times s$ matrix $H$ formed by this table has the property that $H^{*} H=H H^{*}=s I$, where $H^{*}$ is the adjoint of $H$ ( $H$ is thus a complex Hadamard matrix).

Let $G=G_{1} \times \cdots \times G_{k}$ where each $G_{i}$ is an abelian group, so that $G$ is as well, and assume that the elements of $G$ are ordered in some fashion. (Ai and Zhang [1 use a lexicographic or Yates order).) If we consider the set of $J$-characteristics $\chi_{g}(D)$ and the counts $O(g)$ as $s \times 1$ vectors $\chi$ and $O$ indexed by $g \in G$, then (5) may be written

$$
\chi=H O \text {. }
$$

Multiplying through by $H^{*}$, we see that $H^{*} \chi=H^{*} H O=s O$, so that

$$
O=(1 / s) H^{*} \chi
$$

and in particular that the $J$-characteristics determine the design. This is Theorem 1 of [1].

However, in general $H$ depends on the group structure of $G$, and so from (7) or directly from (5) we see that the values of the $J$-characteristics depend on the choice of group structure. This is illustrated with the following example.

Example 3.1. Consider the 3-factor design

$$
D=\left[\begin{array}{cccc}
0000 & a a a a & b b b b & c c c c \\
0 a b c & 0 a b c & 0 a b c & 0 a b c \\
0 a b c & b 0 c a & a c 0 b & c b a 0
\end{array}\right]
$$

Each factor has 4 levels, namely $0, a, b$, and $c$, and each column is a treatment combination. One can check that this is an orthogonal array of strength 2 and index 1 (it is taken from [5], where it is shown to be non-regular).

For each factor the symbol set $G_{i}=\{0, a, b, c\}$ may be given two group structures, namely that of the cyclic group $\mathbb{Z}_{4}$ and that of the "Klein 4-group" $V$ (isomorphic to $\mathbb{Z}_{2} \times \mathbb{Z}_{2}$ ). Table 3.1 displays the non-zero values of $\chi_{g}(D)$ as $g$ runs over the 64 elements of $G=G_{1} \times G_{2} \times G_{3}$, 
Table 1: $J$-Characteristics for Design $D$ under two different group structures For the design in (8), the value of $\chi_{g}(D)$ is given for each $g \in G$, where $G=$ either $\mathbb{Z}_{4} \times \mathbb{Z}_{4} \times \mathbb{Z}_{4}$ or $V \times V \times V$. Those $g$ for which both values of $\chi_{g}(D)=0$ are omitted. (Computation was done in Maple.)

\begin{tabular}{ccccccccccc}
\hline$g=$ & 000 & $a a a$ & $a a b$ & $a a c$ & $a b a$ & $a b b$ & $a b c$ & $a c a$ & $a c b$ & $a c c$ \\
\hline $\mathbb{Z}_{4}$ & 16 & $-6-2 i$ & $4 i$ & $6-2 i$ & $-4 i$ & $4+4 i$ & -4 & $6-2 i$ & 4 & $6+2 i$ \\
$V$ & 16 & 8 & 8 & 0 & -8 & 8 & 0 & 0 & 0 & 0 \\
& & & & & & & & & & \\
\hline$g=$ & $b a a$ & $b a b$ & $b a c$ & $b b a$ & $b b b$ & $b b c$ & $b c a$ & $b c b$ & $b c c$ & \\
\hline $\mathbb{Z}_{4}$ & $4 i$ & $-4-4 i$ & 4 & $4+4 i$ & 8 & $4-4 i$ & 4 & $-4+4 i$ & $-4 i$ & \\
$V$ & 8 & -8 & 0 & 8 & 8 & 0 & 0 & 0 & 0 & \\
& & & & & & & & & & \\
\hline$g=$ & $c a a$ & $c a b$ & $c a c$ & $c b a$ & $c b b$ & $c b c$ & $c c a$ & $c c b$ & $c c c$ & \\
\hline $\mathbb{Z}_{4}$ & $6-2 i$ & 4 & $6+2 i$ & -4 & $4-4 i$ & $4 i$ & $6+2 i$ & $-4 i$ & $-6+2 i$ \\
$V$ & 0 & 0 & 0 & 0 & 0 & 0 & 0 & 0 & 16 & \\
\hline
\end{tabular}

where the groups $G_{i}$ are all $\mathbb{Z}_{4}$ or all $V$. For example, $\chi_{\text {aaa }}(D)=-6-2 i$ using $\mathbb{Z}_{4}$, but $=8$ using $V$. Thus we see that the values of the $J$-characteristics depend on the group structure. Note that for both group structures we have $\chi_{g}(D)=0$ if wt $(g)=1$ or 2 . Such group elements have been omitted from the table for convenience.

It is not hard to calculate the values $A_{j}(D)$ given by equation (6), where we have $N=16$. (The computation is shortened by the fact that $| \pm a \pm b i|^{2}=a^{2}+b^{2}$.) We find that under both group structures we have $A_{1}(D)=A_{2}(D)=0$ and $A_{3}(D)=3$, as guaranteed by Theorem 2.2 ,

Before we leave this topic, we develop the properties of the character table a little further.

In enumerating the elements of a group $G$ one typically chooses $g_{1}=$ the identity. With this convention, which we shall adopt, $\chi_{g_{1}}$ is the trivial character of $G$, so that $\chi_{g_{1}}(h)=1$ for all $h \in G$. On the other hand, since $G$ is abelian, $\chi_{g}\left(g_{1}\right)=1$ for every $g \in G$, and so we see that $H$ must have the form

$$
H=\left(\begin{array}{cccc}
1 & 1 & \cdots & 1 \\
1 & * & \cdots & * \\
\vdots & \vdots & & \vdots \\
1 & * & \cdots & *
\end{array}\right)
$$

The matrix $U=(1 / \sqrt{s}) H$ is said to be the normalized character table of $G$. We list its important properties here, which follow from the preceding.

Lemma 3.2. $U$ unitary $\left(U^{*} U=U U^{*}=I\right)$, and in particular defines an isometry on $\mathbb{C}^{s}$ $(\langle U v, U w\rangle=\langle v, w\rangle)$. If $e=[1,0, \ldots, 0]^{\prime}$ and $b=(1 / \sqrt{s})[1, \ldots, 1]^{\prime}$ then

$$
U e=b=U^{*} e .
$$




\section{Independence of group structure}

By imposing a group structure on the set $G=G_{1} \times \cdots \times G_{k}$, we define the irreducible characters $\chi_{g}$. We want to show that the numbers

$$
\sum_{\operatorname{wt}(g)=j}\left|\chi_{g}(D)\right|^{2}, \quad j=1, \ldots, k
$$

appearing in (6) are independent of the group structure chosen. This sum is somewhat unwieldy, and so we will break it into smaller sums over elements $g$ which are not only of weight $j$ but also differ from the identity in exactly the same components.

To begin with, we fix an order of the elements in each set $G_{i}$, with the understanding that whenever we impose a group structure, the first element will be the identity of the group. We may denote by $1_{i}$ the chosen element of $G_{i}$.

Now, for each $J \subset\{1, \ldots, k\}$ with $|J|=j$, let

$$
S_{J}=\left\{g=\left(g_{1}, \cdots, g_{k}\right) \in G: g_{i} \neq 1_{i} \text { iff } i \in J\right\} .
$$

(Here $J$ is merely an index set and has no relation to the $J$-characteristics mentioned earlier.) Clearly the sets $S_{J}$ are disjoint and their union is the set of elements of $G$ of weight $j$. Then

$$
\sum_{\operatorname{wt}(g)=j}\left|\chi_{g}(D)\right|^{2}=\sum_{|J|=j} \sum_{g \in S_{J}}\left|\chi_{g}(D)\right|^{2} .
$$

We will show that for each $J$ the inner sum

$$
\sum_{g \in S_{J}}\left|\chi_{g}(D)\right|^{2}
$$

is independent of the group structure chosen.

To do this, we will write these sums as squared norms of elements in an appropriate subspace $V_{J}$ of $\mathbb{C}^{s}$. Assuming a fixed ordering of the elements of $G$, the components of a vector $v \in \mathbb{C}^{s}$ are complex numbers indexed by the elements of $G$, something like

$$
v=\left[\cdots, v_{g}, \cdots\right]^{\prime} .
$$

The standard basis elements are of form

$$
e_{g}=[0, \ldots, 0,1,0 \ldots, 0]^{\prime},
$$

where 1 occurs in just the $g$-th coordinate. Then

$$
v=\sum_{g \in G} v_{g} e_{g}
$$

Let

$$
V_{J}=\left\{v=\left[\cdots, v_{g}, \cdots\right]^{\prime} \in \mathbb{C}^{s}: v_{g}=0 \text { if } g \notin S_{J}\right\} .
$$

It is clear that $\operatorname{dim} V_{J}=\left|S_{J}\right|=\prod_{i \in J}\left(s_{i}-1\right)$, and that the sum (10) is $\left\|M_{J}(\chi)\right\|^{2}$ where $M_{J}$ is the orthogonal projection of $\mathbb{C}^{s}$ onto $V_{J}$. Now the next result follows immediately from (7) and the fact that $H=\sqrt{s} U$. 
Proposition 4.1. Suppose that $G=G_{1} \times \cdots \times G_{k}$ where $G_{i}$ is an abelian group. Then the sum in (10) is equal to

$$
s\left\|M_{J} U O\right\|^{2},
$$

where $M_{J}$ is the orthogonal projection of $\mathbb{C}^{s}$ on the subspace $V_{J}, U$ is the normalized character table of $G$, and $O$ is the vector of multiplicities of the design $D$.

Our goal is now to show that the quantity (14) is independent of the group structure of $G$.

A very useful way to describe $V_{J}$ is as follows. Associate to $G_{i}$ the Euclidean space $\mathbb{C}^{s_{i}}$, where the components of a vector $v$ are indexed by the elements of $G_{i}$. Let $e_{g}^{(i)}$ be the unit vector in $\mathbb{C}^{s_{i}}$ having a 1 in the $g$ th place and zeros elsewhere, so that $e_{1}^{(i)}=[1,0, \cdots, 0]^{\prime}$. Define the subspaces $V_{i} \subset \mathbb{C}^{s_{i}}, i=1, \ldots, k$, by setting

$$
\begin{aligned}
V_{i} & =\left(e_{1}^{(i)}\right)^{\perp}, \quad i \in J, \\
& =\operatorname{span}\left(e_{1}^{(i)}\right), \quad i \notin J,
\end{aligned}
$$

where orthocomplement $\left(^{\perp}\right)$ and span are within $\mathbb{C}^{s_{i}}$. Thus the vectors of $V_{i}$ have a zero in the first position if $i \in J$ and zeros in all the other positions if $i \notin J$.

Let $P_{i}$ be the projection of $\mathbb{C}^{s_{i}}$ onto $\operatorname{span}\left(e_{1}^{(i)}\right), I_{i}$ the identity matrix, and $Q_{i}=I_{i}-P_{i}$.

Proposition 4.2. With the above definitions, we have

$$
V_{J}=V_{1} \otimes \cdots \otimes V_{k} .
$$

The orthogonal projection $M_{J}$ of $\mathbb{C}^{s}$ on $V_{J}$ is given by

$$
M_{J}=M_{1} \otimes \cdots \otimes M_{k},
$$

where $M_{i}$ is the orthogonal projection of $C^{s_{i}}$ on $V_{i}$. We have

$$
\begin{aligned}
M_{i} & =Q_{i}, \quad i \in J, \\
& =P_{i}, \quad i \notin J .
\end{aligned}
$$

Proof. The vectors in this tensor product are sums of vectors of the form

$$
v_{1} \otimes \cdots \otimes v_{k}, \quad v_{i} \in V_{i} .
$$

It is not hard to see that a vector of this form has zeros in exactly the positions indexed by $g \notin S_{J}$, so that $V_{1} \otimes \cdots \otimes V_{k} \subset V_{J}$. However,

$$
\begin{aligned}
\operatorname{dim} V_{i} & =s_{i}-1, & & i \in J, \\
& =1 & & \text { otherwise }
\end{aligned}
$$

so $\operatorname{dim} V_{1} \otimes \cdots \otimes V_{k}=\operatorname{dim} V_{1} \cdots \operatorname{dim} V_{k}=\prod_{i \in J}\left(s_{i}-1\right)=\operatorname{dim} V_{J}$. Thus (15) holds, and (16) follows immediately. The formula for $M_{i}$ is obvious.

We also note the following, which is implicit in equation (4)).

Proposition 4.3. If $G_{i}$ is a finite group having character table $H_{i}$ and normalized table $U_{i}$, then $G=G_{1} \times \cdots \times G_{k}$ has character table $H=H_{1} \otimes \cdots \otimes H_{k}$ and normalized character table $U=U_{1} \otimes \cdots \otimes U_{k}$. 
We use this to evaluate the vector $M_{J} U O$ appearing in (14). As in (13), the vector $O$ of multiplicities may be written

$$
O=\sum_{g \in G} O(g) e_{g}
$$

But if $g=\left(g_{1}, \ldots, g_{k}\right) \in G_{1} \times \cdots \times G_{k}$, then

$$
e_{g}=e_{g_{1}}^{(1)} \otimes \cdots \otimes e_{g_{k}}^{(k)}
$$

where $e_{j}^{(i)}$ is the unit vector in $\mathbb{C}^{s_{i}}$ having a 1 in the $j$ th place and zeros elsewhere. Then

$$
O=\sum_{g=\left(g_{1}, \ldots, g_{k}\right)} O(g) e_{g_{1}}^{(1)} \otimes \cdots \otimes e_{g_{k}}^{(k)}
$$

Thus

$$
\begin{aligned}
M_{J} U O & =\sum_{g=\left(g_{1}, \ldots, g_{k}\right)} O(g) M_{J} U\left(e_{g_{1}}^{(1)} \otimes \cdots \otimes e_{g_{k}}^{(k)}\right) \\
& =\sum_{g=\left(g_{1}, \ldots, g_{k}\right)} O(g) M_{1} U_{1}\left(e_{g_{1}}^{(1)}\right) \otimes \cdots \otimes M_{k} U_{k}\left(e_{g_{k}}^{(k)}\right) .
\end{aligned}
$$

To analyze the (squared) norm of this, we need to analyze the terms in such sums. This leads to evaluating $M_{i} U_{i}$ on the basis elements $e_{g_{i}}^{(i)}$. We will just need to do this when $M_{i}=P_{i}$.

Lemma 4.4. For each $i$ and for every $g \in G_{i}$ we have

$$
P_{i} U_{i}\left(e_{g}^{(i)}\right)=\frac{1}{\sqrt{s_{i}}} e_{1}^{(i)} .
$$

Proof. For simplicity, suppress the index $i$. Now for any $w \in \mathbb{C}^{s}$ we have

$$
P w=\left\langle w, e_{1}\right\rangle e_{1},
$$

so from Lemma 3.2 we have

$$
P U v=\left\langle U v, e_{1}\right\rangle e_{1}=\left\langle v, U^{*} e_{1}\right\rangle e_{1}=\langle v, b\rangle e_{1}
$$

for any $v$, with $b=(1 / \sqrt{s})[1, \ldots, 1]^{\prime}$. In particular,

$$
P U\left(e_{g}\right)=\left\langle e_{g}, b\right\rangle e_{1}=\frac{1}{\sqrt{s}} e_{1}
$$

as claimed.

We now evaluate the squared norm of sums of form (17). This will rest on the following calculation. 
Lemma 4.5. Let $I_{j}$ be identity matrix of order $s_{j}$, and let

$$
c_{g_{1} \cdots g_{i}}=\sum_{g_{i+1}, \ldots, g_{k}} O(g)
$$

the sum of the numbers $O(g)$ over those $g \in G$ with the first $i$ values fixed at $\left(g_{1}, \ldots, g_{i}\right)$. Then for every $0 \leq i \leq k$,

$$
\left\|\left(I_{1} \otimes \cdots \otimes I_{i} \otimes P_{i+1} \otimes \cdots \otimes P_{k}\right) U O\right\|^{2}=\frac{1}{s_{i+1} \cdots s_{k}} \sum_{g_{1}, \ldots, g_{i}} c_{g_{1} \cdots g_{i}}^{2} .
$$

In particular, this quantity is independent of the group structure on $G$.

Proof. We see that $\left(I_{1} \otimes \cdots \otimes I_{i} \otimes P_{i+1} \otimes \cdots \otimes P_{k}\right) U O$

$$
\begin{aligned}
& =\sum_{g=\left(g_{1}, \ldots, g_{k}\right)} O(g) I_{1} U_{1}\left(e_{g_{1}}^{(1)}\right) \otimes \cdots \otimes I_{i} U_{i}\left(e_{g_{i}}^{(i)}\right) \otimes P_{i+1} U_{i+1}\left(e_{g_{i+1}}^{(i+1)}\right) \otimes \cdots \otimes P_{k} U_{k}\left(e_{g_{k}}^{(k)}\right) \\
& =\sum_{g=\left(g_{1}, \ldots, g_{k}\right)} O(g) \quad U_{1}\left(e_{g_{1}}^{(1)}\right) \otimes \cdots \otimes U_{i}\left(e_{g_{i}}^{(i)}\right) \otimes \frac{1}{\sqrt{s_{i+1}}} e_{1}^{(i+1)} \otimes \cdots \otimes \frac{1}{\sqrt{s_{k}}} e_{1}^{(k)} \\
& =\frac{1}{\sqrt{s_{i+1} \cdots s_{k}}} \sum_{\left(g_{1}, \ldots, g_{i}\right)}\left(\sum_{\left(g_{i+1}, \ldots, g_{k}\right)} O(g)\right) U_{1}\left(e_{g_{1}}^{(1)}\right) \otimes \cdots \otimes U_{i}\left(e_{g_{i}}^{(i)}\right) \otimes e_{1}^{(i+1)} \otimes \cdots \otimes e_{1}^{(k)} \\
& =\frac{1}{\sqrt{s_{i+1} \cdots s_{k}}} \sum_{\left(g_{1}, \ldots, g_{i}\right)} c_{g_{1} \cdots g_{i}} U_{1}\left(e_{g_{1}}^{(1)}\right) \otimes \cdots \otimes U_{i}\left(e_{g_{i}}^{(i)}\right) \otimes e_{1}^{(i+1)} \otimes \cdots \otimes e_{1}^{(k)} .
\end{aligned}
$$

But for each $j$, the set $\left\{U_{j}\left(e_{g}^{(j)}\right), g \in G_{j}\right\}$ is orthonormal in $\mathbb{C}^{s_{j}}$ as the unit vectors $e_{g}^{(j)}, g \in G_{j}$, are orthonormal and $U_{j}$ is an isometry. Hence the elements $U_{1}\left(e_{g_{1}}^{(1)}\right) \otimes \cdots \otimes U_{i}\left(e_{g_{i}}^{(i)}\right) \otimes e_{1}^{(i+1)} \otimes$ $\cdots \otimes e_{1}^{(k)}$ are orthonormal in $\mathbb{C}^{s}$, and so $\left\|\left(I_{1} \otimes \cdots \otimes I_{i} \otimes P_{i+1} \otimes \cdots \otimes P_{k}\right) U O\right\|^{2}$

$$
=\frac{1}{s_{i+1} \cdots s_{k}} \sum_{\left(g_{1}, \ldots, g_{i}\right)} c_{g_{1} \cdots g_{i}}^{2}\left\|U_{1}\left(e_{g_{1}}^{(1)}\right) \otimes \cdots \otimes U_{i}\left(e_{g_{i}}^{(i)}\right) \otimes e_{1}^{(i+1)} \otimes \cdots \otimes e_{1}^{(k)}\right\|^{2} .
$$

But this

$$
\begin{aligned}
& =\frac{1}{s_{i+1} \cdots s_{k}} \sum_{\left(g_{1}, \ldots, g_{i}\right)} c_{g_{1} \cdots g_{i}}^{2}\left\|U_{1}\left(e_{g_{1}}^{(1)}\right)\right\|^{2} \cdots\left\|U_{i}\left(e_{g_{i}}^{(i)}\right)\right\|^{2}\left\|e_{1}^{(i+1)}\right\|^{2} \cdots\left\|e_{1}^{(k)}\right\|^{2} \\
& =\frac{1}{s_{i+1} \cdots s_{k}} \sum_{\left(g_{1}, \ldots, g_{i}\right)} c_{g_{1} \cdots g_{i}}^{2}
\end{aligned}
$$

as all the norms in the next-to-last line are 1. This is formula (18).

Now fix $J \subset\{1, \ldots, k\}$.

Proposition 4.6. $\left\|M_{J} U O\right\|^{2}$ is independent of the group structure of $G$. 
Proof. Actually, we will prove something more general, namely that the proposition holds for projections $M$ made up of a tensor product of $P_{i}$ 's, $Q_{i}$ 's and $I_{i}$ 's, where $Q_{i}=I_{i}-P_{i}$. Letting $q=$ the number of factors $Q_{i}$ in the projection, we prove this by induction on $q$.

We simplify matters by proving our result for projections of form

$$
M=Q_{1} \otimes \cdots \otimes Q_{q} \otimes I_{q+1} \otimes \cdots \otimes I_{q+i} \otimes P_{q+i+1} \otimes \cdots \otimes P_{k} .
$$

The proof is the same for projections with other ordering of the tensor factors.

The base case $(q=0)$ is precisely Lemma 4.5. For the induction step, assume that the result holds for projections having $q-1$ factors $Q$ (not necessarily the first $q-1$ factors). Now $Q_{q}=I_{q}-P_{q}$, so the projection (19) is

$$
\begin{aligned}
M= & Q_{1} \otimes \cdots \otimes Q_{q-1} \otimes I_{q} \otimes I_{q+1} \otimes \cdots \otimes I_{q+i} \otimes P_{q+i+1} \otimes \cdots \otimes P_{k} \\
& -Q_{1} \otimes \cdots \otimes Q_{q-1} \otimes P_{q} \otimes I_{q+1} \otimes \cdots \otimes I_{q+i} \otimes P_{q+i+1} \otimes \cdots \otimes P_{k} \\
& =T_{1}-T_{2},
\end{aligned}
$$

say. Since $T_{1}=M+T_{2}$ and $M$ and $T_{2}$ are orthogonal, the Pythagorean Theorem gives

$$
\|M U O\|^{2}=\left\|T_{1} U O\right\|^{2}-\left\|T_{2} U O\right\|^{2} .
$$

But since $T_{1}$ and $T_{2}$ contain $q-1$ factors $Q_{i}$, the induction hypothesis applies to both terms on the right-hand-side of (20), and therefore to the left-hand-side, as desired.

By Proposition 4.1 this shows that the sum (10), and therefore the quantities $A_{j}(D)$, are independent of the group structure of $G$. Theorem 2.2 is now proved.

\section{Conclusion}

The definition of the generalized wordlength pattern (GWLP) given in [15] makes sense if one chooses abelian rather than cyclic groups to index the levels of each factor. The choice to use cyclic groups in [15] is arbitrary, and we have shown that while it does affect the so-called $J$-characteristics of a design, it does not affect the GWLP. This removes a possible ambiguity in the definition of the GWLP, and therefore in the use of minimum aberration as an optimality criteria for nonregular designs. The choice of cyclic groups may be useful computationally as the irreducible characters are then especially simple.

A special case of the invariance with respect to group structure is already implicit in the coding literature [3. (The connection with regular designs is given in [15.) However, this covers designs in which (a) the index sets $G_{i}$ are the same (the alphabet) and (b) the design is actually a subset of $G$ (so that the counting function $O$ is simply an indicator function). Our Theorem 2.2 is quite general, and makes no use of concepts borrowed from coding theory.

The wordlength pattern of a regular design does not determine the design, and in particular does not tell us its alias structure. For that, one needs the defining words. We have seen that an analog of the set of defining words of a nonregular design is the set of $J$-characteristics, at least in respect of determining the design. However, as we noted in Section 3 , the $J$-characteristics vary with the choice of group structure assigned to factors. Certainly the aliasing structure of a design does not depend on this arbitrary choice. The GWLP is independent of this choice, and 
one may therefore ask just what statistical information it carries. This is a question worthy of further investigation.

Acknowledgment. We thank Dan Lutter for some useful discussions, and the referee and editor for some helpful suggestions.

\section{References}

[1] Ming-Yao Ai and Run-Chu Zhang. Projection justification of generalized minimum aberration for asymmetrical fractional factorial designs. Metrika, 60:279-285, 2004.

[2] Joel G. Broida and S. Gill Williamson. A Comprehensive Introduction To Linear Algebra. Addison-Wesley Publishing Company Advanced Book Program, Redwood City, CA, 1989.

[3] Philippe Delsarte. Four fundamental parameters of a code and their combinatorial significance. Information and Control, 23:407-438, 1973.

[4] Lih-Yuan Deng and Boxin Tang. Generalized resolution and minimum aberration criteria for Plackett-Burman and other nonregular factorial designs. Statistica Sinica, 9:1071-1082, 1999.

[5] Wiebke S. Diestelkamp and Jay H. Beder. On the decomposition of orthogonal arrays. Utilitas Mathematica, 61:65-86, 2002.

[6] M. F. Franklin. Constructing tables of minimum aberration $p^{n-m}$ designs. Technometrics, 26:225-232, 1984.

[7] A. Fries and W. G. Hunter. Minimum aberration $2^{k-p}$ designs. Technometrics, 22:601-608, 1980.

[8] I. Martin Isaacs. Character Theory of Finite Groups. Academic Press, New York, 1976.

[9] Walter Ledermann. Introduction to Group Characters. Cambridge University Press, Cambridge, 2nd edition, 1987.

[10] Jean-Pierre Serre. Linear Representations of Finite Groups. Springer-Verlag, New York, 1977. Leonard L. Scott, transl.

[11] Akimichi Takemura. Tensor analysis of ANOVA decomposition. Journal of the American Statistical Association, 78:894-900, 1983.

[12] Boxin Tang. Theory of $J$-characteristics for fractional factorial designs and projection justification of minimum $G_{2}$-aberration. Biometrika, 88:401-407, 2001.

[13] Boxin Tang and Lih-Yuan Deng. Minimum $G_{2}$-aberration criteria for nonregular fractional factorial designs. The Annals of Statistics, 9:1914-1926, 1999.

[14] Hongquan Xu. Personal communication. 
[15] Hongquan Xu and C. F. J. Wu. Generalized minimum aberration for asymmetrical fractional factorial designs. The Annals of Statistics, 29:1066-1077, 2001.

\section{A Multilinear background}

In this section we briefly review some results on tensor products that we have used in this paper. We only deal with Euclidean spaces (specifically $\mathbb{C}^{k}$ ) since that is all we need here. For simplicity we concentrate on the bilinear case (two tensor factors).

There are many expositions of multilinear algebra, such as that in [2]. An interesting exposition with some statistical applications is given in [11].

As is well-known, the Kronecker or tensor product of the matrices $A(m \times n)$ and $B$ is

$$
A \otimes B=\left[\begin{array}{ccc}
a_{11} B & \cdots & a_{1 n} B \\
\vdots & \ddots & \vdots \\
a_{m 1} B & \cdots & a_{m n} B
\end{array}\right]
$$

For vectors $v \in \mathbb{C}^{a}$ and $w \in \mathbb{C}^{b}$ we thus have

$$
v \otimes w=\left[\begin{array}{c}
v_{1} w \\
\vdots \\
v_{a} w
\end{array}\right] \in \mathbb{C}^{a b},
$$

where $v=\left[v_{1}, \ldots, v_{a}\right]^{\prime}$. This product satisfies the usual bilinear properties, for example, $c v \otimes$ $w=c(v \otimes w)=v \otimes c w(c$ a scalar $)$ and $A \otimes(B+C)=A \otimes B+A \otimes C$.

If $V \subset \mathbb{C}^{a}$ and $W \subset \mathbb{C}^{b}$ are subspaces, then we define their tensor product to be the subspace of $\mathbb{C}^{a b}$ given by

$$
V \otimes W=\operatorname{span}\{v \otimes w: v \in V, w \in W\} .
$$

(Technically, $V \otimes W$ is constructed as a free vector space modulo bilinear relations, and is only isomorphic to a subspace of $\mathbb{C}^{a b}$, but we will identify it with that subspace.) If $\left\{e_{1}, \cdots, e_{k}\right\}$ is a basis of $V$ and $\left\{f_{1}, \cdots, f_{\ell}\right\}$ is a basis of $W$, then

$$
\left\{e_{i} \otimes f_{j}: i=1, \ldots k, j=1, \ldots \ell\right\}
$$

is a basis of $V \otimes W$. Thus in particular

$$
\operatorname{dim}(V \otimes W)=\operatorname{dim}(V) \cdot \operatorname{dim}(W) .
$$

If we use $\left\langle v_{1}, v_{2}\right\rangle$ to denote the inner (or dot) product and $\|v\|=\sqrt{\langle v, v\rangle}$ the norm, then we have

$$
\left\langle v_{1} \otimes w_{1}, v_{2} \otimes w_{2}\right\rangle=\left\langle v_{1}, v_{2}\right\rangle\left\langle w_{1}, w_{2}\right\rangle
$$

and in particular

$$
\|v \otimes w\|=\|v\|\|w\|
$$


The norm and inner product of vectors of the form $\sum v_{i} \otimes w_{i}$ are calculated by expanding the inner product in the usual way.

If $T_{i}$ is a linear transformation on $V_{i}$, then $T=T_{1} \otimes T_{2}$ is a linear transformation on $V_{1} \otimes V_{2}$ such that

$$
T\left(v_{1} \otimes v_{2}\right)=T_{1}\left(v_{1}\right) \otimes T_{2}\left(v_{2}\right) .
$$

$T$ is evaluated on sums of such terms by linearity. The matrix of $T$ is given by the Kronecker product of the matrices $T_{i}$. Finally, if $S=S_{1} \otimes S_{2}$ is a linear transformation such that $S_{i} T_{i}$ is defined for each $i$, then

$$
S T=S_{1} T_{1} \otimes S_{2} T_{2}
$$

All of the preceding extends in the obvious way to more than two tensor factors. 